\title{
Fluoroquinolones: Utilisation and Compliance with WHO Defined Daily Doses
}

\author{
Mohd Alfam Shamin Ashraf Alisha1, Abdullah Khan1,*, Ching Siang Tan1, Shashidharan Menon', Jaasminerjiit Kaur', \\ Anandarajagopal Kalusalingam ${ }^{1}$, Mei Jun Loy ${ }^{2}$, Mohamed Mansor Manan ${ }^{3}$, Long Chiau Ming ${ }^{4}$ \\ 'School of Pharmacy, KPJ Healthcare University College, Nilai, MALAYSIA. \\ ${ }^{2}$ Faculty of Engineering, Universiti Teknologi Malaysia, Johor, MALAYSIA. \\ ${ }^{3}$ Faculty of Pharmacy, Universiti Teknologi MARA, Puncak Alam, Selangor, MALAYSIA \\ ${ }^{4}$ PAPRSB Institute of Health Sciences, Universiti Brunei Darussalam, Gadong, BRUNEI DARUSSALAM.
}

\begin{abstract}
Background: Fluoroquinolones are indicated for the treatment of a number of infections due to their broad-spectrum activity. Objectives: In the present study we aimed to describe the types and characteristics of fluoroquinolones prescribed, to examine the pattern of Fluoroquinolones used and to calculate and compare the Defined Daily Doses (DDD) for each Fluoroquinolones with the WHO guidelines. Methods: The full prescription record of patients prescribed with fluoroquinolones was reviewed and the data which met the inclusion and exclusion criteria were recorded. In this study, the data were recorded as the DDD per 1000 inhabitants per day. Data from the patient's prescription record and demographic profile were assessed using descriptive statistics. Results and Discussion: Out of 200 screened patients' prescription, 51\% were males. Patient in the age group of $>60$ years old was $30.5 \%$; followed by age group $40-49$ years, $18.5 \%$. Ciprofloxacin $250 \mathrm{mg}$ (Ciprobay $250 \mathrm{mg}$ ) was the most widely prescribed fluoroquinolone, accounting for $31 \%$ of the prescriptions. This was followed by levofloxacin 500mg (Cravit 500mg) $25 \%$ and moxifloxacin 400mg (Avelox 400mg) 18.5\%. Ofloxacin (Tarivid 100mg) was only $4 \%$. In
\end{abstract}

term of calculation based on DDD, the four most utilized fluoroquinolones were levofloxacin 500mg (0.049 DID) followed by moxifloxacin (0.03 DID), Ciprofloxacin 500mg (0.028 DID), and ciprofloxacin 250mg (0.024 DID). The least utilized antibiotic was ofloxacin $100 \mathrm{mg}$ (0.0069 DID). The total fluoroquinolones antibiotics used for the outpatient department in the hospital were 0.1409 DID. Conclusion: The accessed data revealed that the DDD, for all the Fluoroquinolones utilized at this hospital complied with the recommended WHO DDD.

Key words: Pharmacoepidemiology, Antimicrobial, Ciprofloxacin, Levofloxacin, Prescribing pattern, DDD.

\section{Correspondence}

Dr. Abdullah Khan

School of Pharmacy, KPJ Healthcare University College, Nilai, MALAYSIA.

Email: abdullahkhan@kpjuc.edu.my

DOI: 10.5530/jyp.2021.13.28

\section{INTRODUCTION}

Prescribing practices can be defined as the ability of health professionals to differentiate and discriminate among the several choices of drugs and to determine the ones that will be the most beneficial to their patients. ${ }^{1}$ The study on the prescribing pattern is one of the requirements of the medical audit such as Joint Commission Standards, where it seeks to evaluate, monitor, and suggest possible modifications in prescribing practices to make medical care rational and cost-effective. ${ }^{2}$ Drug Utilization Review (DUR) is defined by the World Health Organization (WHO) as, the marketing, distribution, prescription and use of drugs in a society, with special emphasis on the resulting medical, social, and economic consequences.

According to WHO, DDD is the assumed average maintenance dose per day for a drug used for its main indication in adults. ${ }^{3}$ It should be clearly understood that DDD is a measurement unit and does not relate to the prescribed daily dose (PDD). Some patients may have a different dose from the recommended DDD due to the difference in some characteristics such as their age, weight, and pharmacokinetic considerations. DDD is based on the available information about doses from various countries and the doses which are usually being prescribed. DUR should be ideally presented as numbers of DDDs per 1000 inhabitants per day if it is used in the outpatient and DDDs per 100 bed-days if it is used in inpatient. As for anti-infective drugs such as antibiotics, the most proper way to present the Figures are by DDDs per inhabitant per year. ${ }^{4}$
An antibiotic is a constituent produced by one microorganism which selectively inhibits the growth of another. In an economically developing nation, boundless empirical prescribing of antibiotics exists, and it is a concern since improper use of antibiotics will increase the resistance rates, this is supported by a report from Malaysia Statistic on Medicine 2010. ${ }^{5}$ Thus, there is a need to evaluate the antibiotic utilization across healthcare sectors to improve future use and prescribing patterns and to prevent any adverse event. This includes the fluoroquinolones which are widely used among hospitals, including those in Malaysia. Fluoroquinolones are used in the treatment of several infections due to their broad-spectrum activity against most Gram-positive and Gramnegative bacteria which are often misused or over-used. ${ }^{6,7}$

Irrational antimicrobial use is a global problem, especially in economically developing nations. It often fails to destroy the infectious microbes, the emergence of AMR, as well as unnecessary healthcare costs to the patients and healthcare systems. ${ }^{8}$ AMR may increase the difficulty in treating the patients with standard first-line antibiotics and may lead to the use of newer and more expensive antibiotics across healthcare sectors. In Malaysia, a study by Rahman et al. on comparison of prescribing antibiotics in public and private practice in primary clinics reveals an uncontrolled antibiotic usage, especially in private sectors. ${ }^{9}$

There are only a few studies related to DUR on antimicrobial agents in the hospital setting. In Malaysia, only a few studies have been carried 
out to evaluate the antibiotic utilization pattern, despite the obvious increase in fluoroquinolone resistance during the past years as reported by Malaysia Antibiotic Resistance Surveillance. Therefore, the evaluation of the use of antibiotics in Malaysia is an essential measure for assessing and improving the appropriate use of antibiotics across sectors to develop proper future strategies to reduce AMR as well as to minimize the risk of morbidity and mortality due to AMR which are currently increasing in our country. In Malaysia, according to the Malaysia Antibiotic Resistance Surveillance, there is an increase in fluoroquinolones resistance in 2016 compared to 2015, especially on Salmonella typhi spp. that is from $1.5 \%$ in 2015 to $5.2 \%$ in $2016 .{ }^{10}$ This increase in the resistance to antibiotics necessitates the evaluation of antibiotic usage particularly in the private healthcare sector and to promote the rational use of antibiotics.

This study was aimed to describe DUR of fluoroquinolones in an outpatient setting in a private hospital in the Klang Valley area. Furthermore, prescribing pattern of fluoroquinolones, the compliance of DDD for fluoroquinolones antibiotics with WHO recommendations, as well as the cost per prescription for a patient who treated with fluoroquinolones antibiotics, were also determined.

Information regarding the utilization and the appropriateness in prescribing the fluoroquinolones in private hospital settings were determined and, this assessment will help to deter irrational antibiotics prescribing as well as unnecessary healthcare costs to patients and healthcare systems. The results will also be useful to improve or review current policy on the utilization of antibiotics. The findings will be used to inform the healthcare providers on antibiotic use in private healthcare settings.

\section{MATERIALS AND METHODS}

This study was a retrospective study on the utilization of fluoroquinolones antibiotic conducted in the outpatient department of a private healthcare centre in Klang Valley, Malaysia. This was a retrospective observational study using convenience sampling. The data was conducted for one year from January 2017 to December 2017, and the accessed data was compiled in February 2018.

A retrospective study is a study that looks backward while an observational study is usually based on inferences drawn from a sample to a population where the independent variable is not under the control of the researcher. As for the convenience sampling method, it is a non-probability sampling method that relies on data collection from population members readily and conveniently available to participate in the study (Figure 1).

\section{Prescription and Patient Record Selection Criteria}

The samples were randomly selected from the outpatient poison list book every month from January 2017 to December 2017. The demography and medication record of the patients listed in the prescription was then collected from the hospital EMR. The selection of prescription and its patient profile was based on the following inclusion and exclusion criteria. ${ }^{11}$ Patients of age groups eighteen years, receiving oral fluoroquinolones in outpatient settings, medication records are available in the EMR are included in the study and a prescription from other institutions and Fluoroquinolones for topical use are excluded.

\section{Sampling Procedure}

The list of all fluoroquinolone antibiotics was identified based on the hospital's drug formulary. The list of the patient taking fluoroquinolone antibiotics was then obtained from outpatient poison list book. The patients were selected by convenient sampling technique every month from January 2017 to December 2017. Subsequently, using the patient Medical Record Number (MRN) the full prescription was obtained
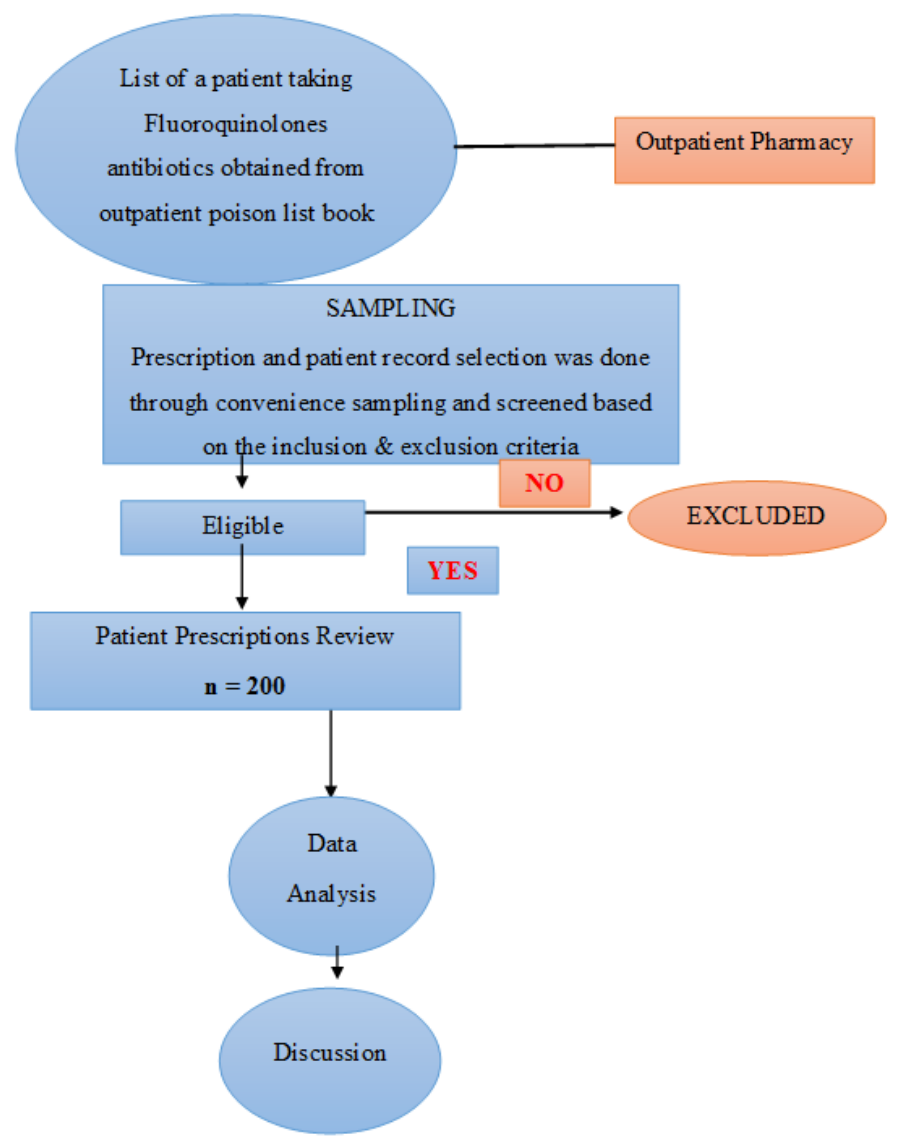

Figure 1: Patient Selection Flowchart.

from the KCIS system and the data meeting the inclusion and exclusion criteria were collected.

A data collection form was used in gathering patients' information. Information on patient demographic were collected. The dependent variables included were DDD of fluoroquinolones, direct drug cost and prescribing pattern, while the independent variables included are medication dosage and average fluoroquinolones use per day. Information on the prescribed medications, dose and frequency were also recorded individually, and the DDD of fluoroquinolones antibiotics per 1,000 inhabitants per day were calculated.

\section{Sample Study and Ethical Consideration}

The sample size required for this study was calculated based on a formula proposed by Naing. ${ }^{12}$ The minimum sample size calculated was 100 patients, $20 \%$ additional sample was added to prevent any data loss. Thus, the sample size was inflated to 120 patients. Ethical issues such as confidentiality and informed consent were considered in this study. This study was approved by the Research Ethics Committee of KPJ Healthcare University College, Nilai, Malaysia. Ethical clearance approval number: KPJUC/RMC/BPH/EC/2017/9.

\section{Outcome Parameter}

The data on demographic variables influencing the antibiotic prescribing were collected and analyzed such as age, gender, race, and duration of treatment which is obtained from the Hospital EMR. The fluoroquinolones antibiotics prescribed, DDD per thousand inhabitants daily were also recorded to assess the prescribing pattern and appropriateness of prescribing. The DDD calculated was then compared 
with WHO classification of ATC/DDD obtained from ATC/DDD Index 2017 provided on the webpage of WHOCC.

Types of Fluoroquinolones and its ATC CODE which have been registered in Malaysia and available in the private healthcare settings were Ofloxacin (J01MA01), Ciprofloxacin (J01MA02), Levofloxacin (J01MA12), and Moxifloxacin (J01MA14).

\section{Statistical Analysis}

Demographic information and prescribing record of each patient was analyzed descriptively by using the Statistical Package for Social Science (SPSS) program version 22.0. The data are expressed as mean and standard deviation. Data of descriptive statistics in terms of frequencies and percentages and presented in the form of graphs, Tables, and charts.

\section{RESULTS}

The prescription data obtained from the respective hospital setting were selected based on the defined criteria. Data that have met all the inclusion criteria were critically analyzed. From the total number of outpatient prescriptions in 2017, 228 prescriptions were selected based on the inclusion and exclusion criteria. However, due to some incomplete data in a few of the selected prescription, only 200 prescriptions were used for the final analysis. The following evaluation was made from the data.

\section{Patients' Socio-Demographic Characteristics}

A total of 200 outpatient prescriptions were included in the study. Out of the 200 patients, $102(51 \%)$ were males, and the balance 98 (49\%) are female patients. As for the age distribution, most of the patients 61 (30.5\%) were in the age group of $>60$ years old followed by 37 (18.5\%) in the age group 40-49 years. The age-wise distribution of the patients is presented in Table 1. The demographic analysis showed that Malay patients were highest with 107 (53.5\%) to receive fluoroquinolone antibiotics followed by Chinese 64 (32\%) and Indians18 (9\%). The remaining were others, including the foreigners, Sikhs, and the Sabah and Sarawak Bhumiputra.

\section{Type and Education Background of the Prescribers}

From the data it was evident that most prescribers (142) that is $71 \%$ were Specialists while the rest (58) 29\% Medical Officers. Out of 142 prescriptions being prescribed by the specialist, around 135 (95\%) of the specialist were overseas graduates while the other (7) $5 \%$ were graduates from local Malaysian universities.

Table 1: Patients' Socio-demographic characteristics.

\begin{tabular}{|c|c|c|c|}
\hline \multirow{2}{*}{\multicolumn{2}{|c|}{$\begin{array}{c}\text { Demographic variables } \\
\mathrm{N}\end{array}$}} & \multicolumn{2}{|c|}{ No and Percentage of Prescription } \\
\hline & & Percentac & \\
\hline \multirow{2}{*}{ Gender } & Male & 102 & 51 \\
\hline & Female & 98 & 49 \\
\hline \multirow{6}{*}{ Age (years) } & $<20$ & 3 & 1.5 \\
\hline & $20-29$ & 34 & 17 \\
\hline & 30-39 & 29 & 14.5 \\
\hline & $40-49$ & 37 & 18.5 \\
\hline & 50-59 & 36 & 18 \\
\hline & $>60$ & 61 & 30.5 \\
\hline \multirow{4}{*}{ Race } & Malay & 107 & 53.5 \\
\hline & Indian & 18 & 9 \\
\hline & Chinese & 64 & 32 \\
\hline & Others & 11 & 5.5 \\
\hline
\end{tabular}

\section{Prescription Pattern of Fluoroquinolones}

Fluoroquinolones were prescribed by the brand names only. Out of 200 prescriptions collected, Ciprofloxacin 250mg (Ciprobay 250mg) was the most widely prescribed fluoroquinolones, accounting for $31 \%$ of the prescriptions followed by Levofloxacin 500mg (Cravit 500mg) $25 \%$ and Moxifloxacin 400mg (Avelox 400mg) 18.5\%. Ofloxacin (Tarivid 100mg) was only $4 \%$. All fluoroquinolones prescribed were oral formulations in a tablet form.

\section{Type of Infection and Antimicrobial Prescribed}

Highest percentage (47.5\%), of Fluoroquinolones prescription were for respiratory infections followed by gastrointestinal infections $(25.5 \%)$ and genitourinary infection (22.5\%). Details on other types of infection and the antimicrobials prescribed are presented in Table 2.

Table 2: Type of infection and the antimicrobials prescribed.

\begin{tabular}{|c|c|c|c|}
\hline \multirow[t]{2}{*}{$\begin{array}{l}\text { Type of } \\
\text { infection }\end{array}$} & \multirow[t]{2}{*}{ Fluoroquinolones prescribed } & \multicolumn{2}{|c|}{$\begin{array}{l}\text { No and Percentage } \\
\text { of Prescription }\end{array}$} \\
\hline & & $\mathbf{n}$ & Percentage \% \\
\hline \multirow[t]{4}{*}{ Gastrointestinal } & $\begin{array}{l}\text { Ciprofloxacin 250mg (Ciprobay } \\
250 \mathrm{mg} \text { ) }\end{array}$ & 33 & 16.5 \\
\hline & $\begin{array}{c}\text { Ciprofloxacin 500mg (Ciprobay } \\
500 \mathrm{mg} \text { ) }\end{array}$ & 16 & 8 \\
\hline & $\begin{array}{l}\text { Levofloxacin 500mg (Cravit } \\
500 \mathrm{mg} \text { ) }\end{array}$ & 1 & 0.5 \\
\hline & $\begin{array}{l}\text { Moxifloxacin 400mg (Avelox } \\
400 \mathrm{mg} \text { ) }\end{array}$ & 1 & 0.5 \\
\hline \multirow[t]{6}{*}{ Genitourinary } & $\begin{array}{c}\text { Ciprofloxacin 250mg (Ciprobay } \\
250 \mathrm{mg})\end{array}$ & 23 & 11.5 \\
\hline & $\begin{array}{c}\text { Ciprofloxacin 500mg (Ciprobay } \\
500 \mathrm{mg} \text { ) }\end{array}$ & 9 & 4.5 \\
\hline & $\begin{array}{l}\text { Levofloxacin } 250 \mathrm{mg} \text { (Cravit } \\
250 \mathrm{mg} \text { ) }\end{array}$ & 2 & 1 \\
\hline & $\begin{array}{l}\text { Levofloxacin 500mg (Cravit } \\
500 \mathrm{mg} \text { ) }\end{array}$ & 3 & 1.5 \\
\hline & $\begin{array}{l}\text { Moxifloxacin 400mg (Avelox } \\
400 \mathrm{mg} \text { ) }\end{array}$ & 1 & 0.5 \\
\hline & $\begin{array}{l}\text { Ofloxacin } 100 \mathrm{mg} \text { (Tarivid } \\
100 \mathrm{mg} \text { ) }\end{array}$ & 7 & 3.5 \\
\hline \multirow[t]{6}{*}{ Respiratory } & $\begin{array}{c}\text { Ciprofloxacin 250mg (Ciprobay } \\
250 \mathrm{mg})\end{array}$ & 1 & 0.5 \\
\hline & $\begin{array}{c}\text { Ciprofloxacin 500mg (Ciprobay } \\
500 \mathrm{mg} \text { ) }\end{array}$ & 8 & 4 \\
\hline & $\begin{array}{l}\text { Levofloxacin } 250 \mathrm{mg} \text { (Cravit } \\
250 \mathrm{mg} \text { ) }\end{array}$ & 5 & 2.5 \\
\hline & $\begin{array}{l}\text { Levofloxacin 500mg (Cravit } \\
500 \mathrm{mg} \text { ) }\end{array}$ & 46 & 23 \\
\hline & $\begin{array}{l}\text { Moxifloxacin 400mg (Avelox } \\
400 \mathrm{mg} \text { ) }\end{array}$ & 34 & 17 \\
\hline & $\begin{array}{l}\text { Ofloxacin } 100 \mathrm{mg} \text { (Tarivid } \\
100 \mathrm{mg} \text { ) }\end{array}$ & 1 & 0.5 \\
\hline \multirow[t]{3}{*}{$\begin{array}{l}\text { Musculoskeletal } \\
\text { and Skin }\end{array}$} & $\begin{array}{c}\text { Ciprofloxacin 250mg (Ciprobay } \\
250 \mathrm{mg})\end{array}$ & 5 & 2.5 \\
\hline & $\begin{array}{c}\text { Ciprofloxacin 500mg (Ciprobay } \\
500 \mathrm{mg})\end{array}$ & 3 & 1.5 \\
\hline & $\begin{array}{l}\text { Moxifloxacin 400mg (Avelox } \\
400 \mathrm{mg} \text { ) }\end{array}$ & 1 & 0.5 \\
\hline
\end{tabular}




\section{Multiple Prescribing}

Out of 200 prescription collected 182 (91\%) of the prescription containing fluoroquinolones antibiotics were prescribed as monotherapy while the other 18 (9\%) of the prescription fluoroquinolones were prescribed with other anti-infectives. The agents prescribed as monotherapy in large numbers were Ciprofloxacin (43.5\%) followed by Levofloxacin (27.5\%). The commonly prescribed combination drug therapy was Ciprofloxacin + Tinidazole (4.5\%). The details on the utilization of Fluoroquinolones as monotherapy and combination multiple drug therapy are presented in Table 3 and Table 4.

\section{Drug Related Problem}

Out of 200 prescriptions collected, only one prescription was found to have a drug-drug interaction which was between ciprofloxacin and theophylline. All other prescriptions were found to be free of any significant drug- drug interaction. The definition of the term "polypharmacy" used in this study was the use of five or more medications per prescription. Out of 200 prescriptions collected, 12 prescriptions could be classified as polypharmacy.

\section{Total Cost per Prescription}

In term of the total cost per prescription containing fluoroquinolones, most of the prescriptions were in the range of RM100-RM149 (29.5\%) followed by RM150-RM199 (20\%). The mean total cost per prescription was found to be RM 186.21. The details on total cost are presented in Table 5.

\section{Defined Daily Dose}

Total fluoroquinolone antibiotics used for the outpatient department was 0.1409 DID. The four most utilized antibiotics were Levofloxacin $500 \mathrm{mg}$ (0.049 DID) followed by Moxifloxacin (0.03 DID), Ciprofloxacin $500 \mathrm{mg}$ (0.028 DID), and Ciprofloxacin 250mg (0.024 DID). From the results, it was evident that Levofloxacin $500 \mathrm{mg}$ was the most utilized antibiotics in the included samples. The least antibiotics utilized were

\section{Table 3: Fluoroquinolones Prescribed as Monotherapy.}

\begin{tabular}{ccc}
\hline Type of Fluoroquinolones & \multicolumn{2}{c}{ No And Percentage Of Prescription } \\
\cline { 2 - 3 } & N & Percentage \% \\
\hline Ciprofloxacin (Ciprobay) & 87 & 43.5 \\
Levofloxacin (Cravit) & 55 & 27.5 \\
Moxifloxacin (Avelox) & 36 & 18 \\
Ofloxacin (Tarivid) & 4 & 2 \\
\hline
\end{tabular}

Table 4: Fluoroquinolones Prescribed as Combination therapy.

\begin{tabular}{ccc}
\hline & \multicolumn{2}{c}{ No and Percentage of Prescription } \\
\cline { 2 - 3 } Type of Fluoroquinolones & $\mathbf{N}$ & Percentage \% \\
\hline Ciprofloxacin + Tinidazole & 9 & 4.5 \\
Ciprofloxacin + Metronidazole & 1 & 0.5 \\
Ciprofloxacin + Fosfomycin & 1 & 0.5 \\
Levofloxacin + Fosfomycin & 1 & 0.5 \\
Levofloxacin + Metronidazole & 1 & 0.5 \\
Moxifloxacin + Amoxicillin/ & 1 & 0.5 \\
clavulanic acid & 2 & 1 \\
Ofloxacin + Azithromycin + & 2 & 1 \\
Doxycycline & 2 & \\
Ofloxacin + Doxycycline & &
\end{tabular}

Ofloxacin 100mg (0.0069). The details on the Defined Daily Dose are presented in Table 6 and Table 7.

\section{DISCUSSION}

As for the age-wise distribution, it was found that most of the prescriptions were for the age group of $>60$ years old followed by $40-49$ years. This is like a study conducted by ${ }^{13}$ which also found that most of the prescriptions were for an age group of 56-85 years.

Several factors may cause a geriatric patient to become more susceptible to infections. It is a well-known fact that geriatric patients may have comorbidity conditions, such as diabetes, renal insufficiency, and arthritis. Irrespective of the type of comorbidity, it might predispose a person to infections. Other than that, their immune system may not function as would be at their young age. This combination of increased comorbidity and a weakened immune system makes a person more prone to infections. Another syndrome that may also occur when a person becomes old is frailty. Several studies suggest a strong relationship between frailty and disablement. Thus, the risk of falls and injuries increased leading to infections and the need for an antibiotic therapy. ${ }^{14}$

Analysis of the data based on the racial distribution indicated that most of the prescriptions were for Malay ethnic patients followed by Chinese and Indian. The result was found to be similar to the study conducted by ${ }^{11}$ wherein the studies found that most of the prescriptions were prescribed for Malay ethnic. Several factors may affect health differences between ethnic groups. Among these factors are, genetic differences within racial populations, cultural mores, and social and environmental factors. ${ }^{15}$

Analysis of the type of prescribers revealed that the majority of the prescribers were the Specialist. This is similar to a study conducted by, ${ }^{16}$ wherein their study they found that doctors with higher qualification have prescribed more antibiotics compared to those with only a degree qualification. Apart from that in that study also they found that doctors with higher professional qualifications have the right to prescribe more types of antibiotics compared to those with a lower professional qualification. This is similar to the practice in the Malaysia government hospitals, and clinics where the doctor with different qualification having different antibiotic/drug list which they can prescribe.

According to the Malaysia Bluebook Drug Formulary, fluoroquinolone antibiotics were categorized as 'A' for the prescriber category which interprets as the drug which can only be prescribed by the specialist. However, this practice did not apply to the private sector and the result obtained in this study was not influenced by this practice. A slight improvement could be precipitated by the stringent regulations on the use of antimicrobial drugs, and the requirements of using generics such as practiced by the government settings. By doing this it can be ensured that better rational use of drugs can be achieved in the private hospital settings as well.

\section{Table 5: Total Cost per Prescription.}

\begin{tabular}{ccc}
\hline $\begin{array}{c}\text { Total Cost Per } \\
\text { Prescription }\end{array}$ & N & No and Percentage of Prescription \\
\cline { 2 - 3 } & 9 & Percentage \% \\
\hline <RM50 & 20 & 4.5 \\
RM50-RM99 & 59 & 10 \\
RM100-RM149 & 40 & 29.5 \\
RM150-RM199 & 32 & 20 \\
RM200-RM249 & 19 & 16 \\
RM250-RM299 & 21 & 9.5 \\
$>$ RM300 & & 10.5 \\
\hline
\end{tabular}


Table 6: Defined Daily Dose of Fluoroquinolones.

\begin{tabular}{|c|c|c|c|c|c|c|}
\hline Fluoroquinolones & $\begin{array}{l}\text { DDD } \\
(\mathrm{Gm})\end{array}$ & Unit & $\begin{array}{l}\text { Rates per } \\
\text { resident per year } \\
\text { (DDD per } 1000 \\
\text { residents per } \\
\text { day (DID) }\end{array}$ & $\begin{array}{l}\text { Rates per user } \\
\text { per day } \\
\text { (DDD per user } \\
\text { per day) }\end{array}$ & $\begin{array}{c}\text { Intermediate rate } \\
\text { (Days supplied per } \\
\text { user) }\end{array}$ & $\begin{array}{l}\text { Clinical measure } \\
\text { (DDDs/day } \\
\text { supplied) }\end{array}$ \\
\hline Ciprofloxacin $250 \mathrm{mg}$ & 1 & G & 0.024 & 0.192 & 5.77 & $0.5(0.5 \mathrm{gm})$ \\
\hline Ciprofloxacin 500mg & 1 & G & 0.028 & 0.392 & 5.88 & $1(\operatorname{lgm})$ \\
\hline Levofloxacin $250 \mathrm{mg}$ & 0.5 & G & 0.003 & 0.214 & 6.42 & $0.5(0.25 \mathrm{gm})$ \\
\hline Levofloxacin 500mg & 0.5 & G & 0.049 & 0.492 & 7.38 & $1(0.5 \mathrm{gm})$ \\
\hline Moxifloxacin 400mg & 0.4 & G & 0.03 & 0.405 & 6.08 & $1(0.4 \mathrm{gm})$ \\
\hline Ofloxacin 100mg & 0.4 & G & 0.0069 & 0.433 & 6.5 & $1(0.4 \mathrm{gm})$ \\
\hline
\end{tabular}

Table 7: Comparison of WHO Defined Daily Dose and Calculated Defined Daily Dose.

\begin{tabular}{llccccc}
\hline ATC Code & Fluoroquinolones & DDD & Units & Route of administration & $\begin{array}{c}\text { WHO } \\
\text { DDD }\end{array}$ & Units \\
\hline J01MA01 & Ofloxacin 100mg & 0.4 & G & Oral & 0.4 & G \\
J01MA02 & Ciprofloxacin 250mg & 0.5 & G & Oral & Oral & G \\
J01MA02 & Ciprofloxacin 500mg & 1 & G & Oral & 0.5 & G \\
J01MA12 & Levofloxacin 250mg & 0.25 & G & Oral & 0.5 & G \\
J01MA12 & Levofloxacin 500mg & 0.5 & G & Oral & 0.4 & G \\
J01MA14 & Moxifloxacin & 0.4 & G & &
\end{tabular}

Following with the analysis in the relation of education background and the use of fluoroquinolones it was found that from 200 prescriptions, almost all of the prescriptions were prescribed by the doctors with overseas qualification, however to date, there are no published studies conducted on retrospective DUR of fluoroquinolones involving the relation of educational background and the use of fluoroquinolones, hence it was not possible to compare it with other studies, and the findings that were discussed are mainly due to the results obtained.

Analysis of prescription patterns indicated that most of the prescriptions contained ciprofloxacin followed by levofloxacin and moxifloxacin. This is similar to a study conducted by ${ }^{13}$ where, in the study, it was found that out of total prescription of fluoroquinolones prescribed, the highest fluoroquinolone prescribed was ciprofloxacin followed by levofloxacin.

The data showed that ciprofloxacin was used favourably in treating gastroenterology infection, genitourinary infections as well as musculoskeletal and skin infections which account for the most common infections, while most of the levofloxacin prescriptions were for respiratory infections only. Although both fluoroquinolones are in the same group with the same mechanism of action, other factors that might influence the use of ciprofloxacin compared to levofloxacin is the recommendation by the National Antibiotic Guideline 2014 and their effectiveness in treating a particular disease. Most of the drugs in this study were prescribed by the doctors according to the guidelines either as the preferred first-line therapy or alternative.

The non-contributing factor that might also affect the prescribing pattern is the cost factor. The cost might also influence the pattern of prescribing, wherein, in this study it is was found that the cost of oral ciprofloxacin is slightly lower compared to other fluoroquinolone antibiotics thus, this may also be one of the reasons of higher usage of oral ciprofloxacin.
Analysis of the type of infections and the type of antimicrobials prescribed, it was found, that the most common infections treated were respiratory infections. The percentage of fluoroquinolone prescriptions was highest among patients with respiratory infections followed by gastrointestinal infections and genitourinary infections. This is similar to a study conducted in Malaysia by ${ }^{9}$ on antibiotic prescribing in public and private practice, wherein the study it was found that out of total prescription of antibiotics, the most common infection is related to respiratory tract followed by urinary and gastroenterology infection. ${ }^{16}$

Malaysia is one of the South East Asian nations with typical tropical warm and humid weather all year round. West Malaysia faces two monsoon seasons, which are November to March, and late May to September. Thus, the cold weather during the monsoon is one of the reasons for the high rate of respiratory infection in Malaysia. Apart from the slightly lower temperature during monsoon season other factors that may also contribute to high rate of respiratory infection are the air quality, amount of traffic volume and urban land uses which are also potentially affecting the human health concerning the respiratory infection. ${ }^{17}$

From the data of the study, it was found that most of the fluoroquinolones were prescribed as monotherapy. Whereas, only a few of the prescriptions containing fluoroquinolones were prescribed as combination therapy with other anti-infectives. This is almost similar to another study by, ${ }^{13}$ wherein, out of the total prescription of antibiotics prescribed, $87 \%$ of the fluoroquinolone antibiotics were prescribed as monotherapy and $13 \%$ as combination therapy. Although fluoroquinolones are broadspectrum antibiotics, sometimes combination therapy is necessary to achieve efficacy with the use of lower doses. This practice is also useful to achieve additive or synergistic effects, and combat acquired resistance to the antibiotics. 
In this study, it was found that most of the prescriptions containing a combination therapy were intended for the treatment of pouchitis where ciprofloxacin was being prescribed with tinidazole as the choice of treatment. This combination complied with the guideline for the management of pouchitis, where according to a study combination of ciprofloxacin and tinidazole are more effective in the treatment of pouchitis compared with other combinations. ${ }^{18}$

Prescriptions were analysed for drug-drug interaction using The Stockley Drug Interaction Checker 2010. ${ }^{19}$ It was found that one of the prescriptions had a major drug interaction between ciprofloxacin and theophylline. The interaction will cause oral theophylline to increase its level by affecting hepatic/intestinal enzyme CYP1A2 and CYP3A4 metabolism. Of note, this drug interaction was already detected by the pharmacist and communicated to the prescriber involved.

A study by ${ }^{20}$ reported that concomitant use of theophylline and ciprofloxacin could decrease theophylline clearance and increased plasma levels and symptoms of toxicity. Serious and fatal reactions have been also reported which include seizure, status epilepticus, and respiratory failure ${ }^{19}$ Often polypharmacy is referred to as the use of multiple medications, however, there is no consensus on an absolute number of drugs to define polypharmacy. The most common definition for polypharmacy as per the literature is "the concomitant use of five or more drugs and it could enhance drug interactions and drug-related problems". This definition is often controversial, because, polypharmacy may be appropriate to treat a patient with multiple comorbid conditions. This appropriateness is especially true for disease states such as chronic heart failure and diabetes, which require multiple drug therapies as directed by disease state guidelines. ${ }^{21}$ Often multiple co-morbidities are difficult to treat with a smaller number of drugs as each disease require specific drugs for the treatment as well as for prophylaxis, but it is also essential to keep a balance between the number of drugs and effective pharmacotherapy. ${ }^{22}$

As for the polypharmacy for the prescriptions analysed in this study, it was found to have several polypharmacy prescriptions. However, all those prescriptions were found be appropriate based on the patients' condition.

During this study period, it was also found that two patients with the age of 12 and 14 years were prescribed with fluoroquinolones. Research on the safety and efficacy of ciprofloxacin in children in developing countries was reviewed and concluded that ciprofloxacin was safe and efficacious. ${ }^{23}$ However, due to the conflicting findings, it is suggested to be cautious while prescribing fluoroquinolones. The selected hospital does not impose a special restriction on the use of ciprofloxacin among children, the prescribing decision is left to the judgment of the individual physician.

As stated in the objective of the study the total cost per prescription for a patient who is treated with fluoroquinolone antibiotics was also calculated. The findings show that a prescription that contains fluoroquinolones mostly is in the range between RM100-RM149. Furthermore, the mean total cost per prescription containing fluoroquinolones is RM 186.21.

Most of the patients were prescribed with antibiotics based on presumptive diagnosis and clinical skills. All the patients were prescribed with brand-name/innovator products. It is well known that the use of a generic drug may reduce direct drug expenditure owing to their low cost. To date, there are no published studies conducted on retrospective DUR of fluoroquinolones involving total cost per prescription in Malaysia, hence it was not possible to do a comparison between studies.

The total fluoroquinolone antibiotics used for the outpatient department in the study were 0.1409 DID. This is lower than reported by the
Malaysian Statistic 2009 and $2010 .^{24}$ Prescription data presented in DDDs per 1000 inhabitants per day may provide a rough estimation of the proportion of the study samples treated daily with a particular drug or group of drugs. Alternatively, this can be expressed as $0.1409 / 1000$ $(0.014 \%)$ of the samples receive this drug each day in that particular year (2017).

As for the Defined Daily Doses of Fluoroquinolones, the data of the study was compared with the Defined Daily Doses indexed in the WHO Collaborating Centre for Drug Statistics Methodology (ATC/ DDD Index 2013). It was found that the Defined Daily Doses, for all the class of Fluoroquinolones complied with the recommend WHO DDD. It is interesting to note that the two exceptions were for Ciprofloxacin 250mg and Levofloxacin 250mg. However, these doses were complied with the recommended doses by the National Antibiotic Guideline 2014. In this case, the Defined Daily Doses of Ciprofloxacin $250 \mathrm{mg}$ for oral route obtained was $0.5 \mathrm{G}$ and Levofloxacin $250 \mathrm{mg}$ was $0.25 \mathrm{G}$ whereas the recommended WHO Defined Daily Dose of Ciprofloxacin for the oral route are $1 \mathrm{G}$ and Levofloxacin $0.5 \mathrm{G}$ respectively. This indicates that the particular drugs were used for a different indication such as surgical prophylaxis, renal dosing adjustment or smaller size people. Overall it can be said that the usage of fluoroquinolones in the studied hospital is relatively low and complies with the $\mathrm{WHO}$ and National guidelines on the use of antibiotics.

\section{CONCLUSION}

From the detailed analysis of the prescriptions with fluoroquinolone antibiotics, it can be concluded that the DDD obtained complied with the recommended World Health Organization DDD and the usage of fluoroquinolone antibiotics was found to be relatively low. As for the prescribing pattern, it was found that ciprofloxacin is the most frequently prescribed fluoroquinolone. The ongoing antimicrobial stewardship program has already yielded a positive effect on the controlled usage of ciprofloxacin as part of the effort to reduce ciprofloxacin-resistance by the National Antibiotic Resistance Surveillance 2016. The collaboration between pharmacists and prescribers could further strengthen to continue with the safe prescribing of antibiotics. Continuous medical education is a good method to constantly updating the prescriber with all the treatment guidelines and procedures.

\section{Limitation to the Study}

There were a few limitations to the study. Mainly the duration of data collection. Secondly, very limited information can be extracted from the KPJ Clinical Information System and a limitation to assess patient medical records causes the inability to collect and gather other relevant data needed for this study.

\section{ACKNOWLEDGEMENT}

The authors are grateful to the management of KPJ Healthcare University College and the Private Hospital Management for providing the essential information and facilities for this study.

\section{CONFLICT OF INTEREST}

The authors declare no conflict of interest.

\section{ABBREVIATIONS}

ANOVA: Analysis of Variance; AMCP: Academy of Managed Care Pharmacy; AMR: Antimicrobial Resistance; AMS: Antimicrobial Stewardship; ATC: Anatomical Therapeutic Classification; ATC/DDD: Anatomical Therapeutic Classification/ DDD; BMI: Body Mass Index; CI: Confidence interval; DDD: Defined Daily Dose; DID: DDD/1000 
population/day; DNA: Deoxyribonucleic Acid; DUNAS: Dasar Ubat Nasional; DUR: Drug Utilization Review; EPhMRA: European Pharmaceutical Market Research Association; EMR: Electronic Medical Record; Gm: Gram; HITS: Hospital Information Technology System; IMS: Intercontinental Medical Statistics; IM: Intramuscular; IV: Intravenous; KCIS: KPJ Clinical Information System; Kg: Kilogram; MDC: Malaysia Drug Code; Mg: Miligram; MNMP: Malaysian National Medicines Policy; MRN: Medical Record Number; MOH: Ministry Of Health; NAG: National Antibiotic Guidelines; NIACC: National Infection and Antimicrobial Control Committee; NMCS: National Medical Care Survey; PDD: Prescribed Daily Dose; SPSS: Statistical Package for the Social Sciences; TB: Tuberculosis; WHO: World Health Organization.

\section{REFERENCES}

1. Crockett A. Use of Prescription drugs: Rising or declining. Nurs Clin North Am. 2005:40(1):33-49.

2. Shankar PR, Upadhyay DK, Subish P, Dubey AK, Mishra P. Prescribing patterns among paediatric inpatients in a teaching hospital in western Nepal. Singapore Med J. 2006;47(4):261-5.

3. WHO. Introduction to Drug Utilization Research. 2017. http://appswhoint/ medicinedocs/en/d/Js4876e/2html

4. Sjöqvist F, Birkett D. WHO booklet introduction to drug utilization research chapter 10 drug utilization. 2003;76-84.

5. Fauziah SA, Kamarudin A, Aklima NNO. Malaysian Statistics on Medicines 2009 and 2010: Pharmaceutical Services Division and the Clinical Research Centre Ministry of Health. 2014

6. Sharma D, Patel RP, Zaidi ST, Sarker M, Rahman M, Lean QY, et al. Interplay of the quality of ciprofloxacin and antibiotic resistance in developing countries. Frontiers in Pharmacol. 2017;8:546.

7. Kotwani A, Holloway K. Trends in antibiotic use among outpatients in New Delhi India. BMC Infect Dis. 2011;11(1):99.

8. Almalki ZS, Alahmari AK, Guo JJ, Cavanaugh TM. Off-label use of oral fluoroquinolone antibiotics in outpatient settings in the United States, 2006 to 2012. Pharmacoepidemiol Drug Saf. 2016;25(9):1042-51.
9. Rahman NA, Teng CL, Sivasampu S. Antibiotic prescribing in public and private practice: A cross-sectional study in primary care clinics in Malaysia. BMC Infect Dis 2016;16(1):208.

10. National Antibiotic Resistance Surveillance Institute for Medical Research Ministry of Health Malaysia: Institute for Medical Research; 2016.

11. Shamsuddin S. Utilization of antibiotics in primary healthcare setting Universiti Teknologi Mara. 2015.

12. Naing L, Winn T, Rusli BN. Practical issues in calculating the sample size for prevalence studies. Arch Orofac Sci. 2006;1:9-14.

13. Juno JJ, Muneerudeen J, Shastry CS. Drug utilization study of fluoroquinolone antibiotics in a university teaching hospital. J Drug Deliv Ther. 2013;3(6):54-6.

14. Keith SK. Comorbidities metabolic changes make elderly more susceptible to infection. Infec Dis News. 2011. https://wwwhealiocom/infectiousdisease/news/print/infectious-disease-news/\% 7Ba029cda7-ca04-4b1e-98ae677 d27670ceb\% 7D/comorbidities-metabolic-changes-make-elderly-moresusceptible-to-infection

15. Jones SR. Infections in frail and vulnerable elderly patients. Am J Med. 1990;88(3):30S-3S, 38S-42S.

16. Wang H, Li N, Zhu H, Xu S, Lu H, Feng Z. Prescription pattern and its influencing factors in Chinese county hospitals: A retrospective cross-sectional study. PloS One. 2013;8(5):e63225.

17. Ling $\mathrm{OH}$, Musthafa $\mathrm{SN}$, Rasam AR. Urban environmental Health: respiratory infection and urban factors in urban growth corridor of Petaling Jaya, Shah Alam and Klang, Malaysia. Sains Malaysiana. 2014;43:1405-14.

18. Shen B, Lashner BA. Diagnosis and treatment of pouchitis. Gastroenterol Hepatol. 2008;4(5):355-61.

19. Baxter K. Stockley's Drug Interactions Pocket Companion 2010. Pharmaceutical Press: London. UK; 2010.

20. Batty KT, Davis TM, Ilett KF, Dusci LJ, Langton SR. The effect of ciprofloxacin on theophylline pharmacokinetics in healthy subjects. British J Clin Pharmacol. 1995;39(3):305-11.

21. Shah BM, Hajiar ER. Polypharmacy adverse drug reactions and geriatric syndromes. Clin Geriatr Med. 2012;28(2):173-86.

22. Singh N, Yu VL. Rational empiric antibiotic prescription in the ICU. Chest. 2000;117(5):1496-9.

23. Green S, Tillotson G. Use of ciprofloxacin in developing countries. Pediatr Infect Dis J. 1997;16(1):150-9.

24. Fauziah S, Kamarudin ANN. Malaysian statistics on medicines 2009 and 2010. In Centre PSDATCR (ed). Ministry Of Health Malaysia: Petaling Jaya Selangor Malaysia; 2014 